\title{
Plantes, parasites et pathologistes : de la compréhension des interactions à la gestion durable des résistances
}

\section{Didier Andrivon}

Inra

Agrocampus Ouest

Université Rennes 1

UMR 1099 BiO3P (Biologie des organismes et des populations appliquée à la protection des plantes)

Domaine de la Motte, BP 35327

F-35653 Le Rheu Cedex, France

$<$ Didier.Andrivon@rennes.inra.fr>
Tirés à part : D. Andrivon

\begin{abstract}
Résumé
La résistance des plantes aux bioagresseurs, trait génétiquement héritable et donc sélectionnable, fait depuis plusieurs décennies l'objet de recherches approfondies. Si les progrès sont évidents sur le plan de la connaissance - clonage de gènes de résistance et de facteurs d'avirulence, transformation génétique, sélection assistée par marqueurs, construction d'idéotypes variétaux, etc. -, ils se traduisent trop rarement par la mise en ouvre de solutions concrètes sur le terrain. À partir d'une étude de cas sur le mildiou de la pomme de terre, cet article analyse trois éléments clés expliquant ce décalage : 1) la connaissance acquise sur les possibilités d'intégration des plantes résistantes dans des systèmes de protection intégrée reste insuffisante ; 2) les résistances disponibles correspondent mal aux attentes des utilisateurs ; 3) la communication sur les possibilités d'emploi des résistances ne cible pas les véritables décideurs. De cette analyse découlent des propositions pour réconcilier agendas de recherche et exploitation durable des résistances.
\end{abstract}

Mots clés : durabilité ; mildiou oidium ; protection intégrée ; résistance aux maladies.

Thèmes : amélioration génétique ; pathologie ; productions végétales.

\section{Abstract}

Plants, parasites and pathologists: from understanding interaction to sustainable management of resistance

Plant resistance to pests and pathogens, which has been known for over a century to be a heritable - and hence selectable - trait, has been the topic of in-depth research over the past decades. Obvious progress has been made on the detailed knowledge of resistance genes and mechanisms (gene mapping and cloning, genetic transformation, marker-assisted breeding), as well as on a better deciphering of adaptive processes in pest and pathogen populations in response to the use of resistant hosts. Such work has hardly resulted, however, in the widespread adoption of resistant cultivars and/or in substantial increases of resistance durability. On the basis of a case study about potato late blight, this paper attempts to elucidate some of the reasons for this gap, and to offer hints with a view to better integration of available knowledge and an improved understanding of the actors' needs.

Key words: disease resistance; integrated protection; mildiews; sustainability.

Subjects: genetic improvement; pathology; vegetal productions.

T rois groupes d'acteurs principaux doivent être pris en compte pour raisonner la protection des plantes : la plante elle-même ; le (ou les) bioagresseurs qui l'affectent et réduisent par là même son rendement, sa qualité ou le profit économique que le producteur en retire; enfin, le phytiatre (au sens large, incluant le producteur lui-même, les techniciens et conseillers, mais aussi les chercheurs), actuellement sur la sellette vis-à-vis de l'opinion publique. Comme l'ont montré les débats conduits lors du récent Grenelle de l'environnement, et, pour pasticher Sergio Leone, ce trio peut être comparé dans l'esprit de nombre de 
nos concitoyens au bon (qui nourrit les gens et remplit les portefeuilles), à la brute (qui détruit et coûte cher) et au truand (qui traite trop, pollue, sélectionne des OGM dont le consommateur - au moins celui de la plupart des pays développés - ne veut pas, voire travaille des décennies sur des maladies ou des ravageurs sans résoudre véritablement le problème). Comme je fais professionnellement partie de la dernière catégorie, il me semble important de réfléchir sur les raisons pour lesquelles des recherches, entreprises dans le but explicite d'apporter des solutions réalistes à des enjeux majeurs (par exemple réduire de manière massive l'emploi des pesticides sans pour autant compromettre la sécurité alimentaire et la compétitivité des filières agricoles) et menées jusqu'à ce que de telles solutions, validées scientifiquement, soient obtenues, se trouvent trop souvent alimenter un sentiment de défiance visà-vis des chercheurs, chez le contribuable moyen, et d'échec, chez les chercheurs eux-mêmes.

Cet article va donc tenter d'analyser certaines des relations unissant les trois groupes d'acteurs cités plus haut, et de comprendre les freins à l'adoption de méthodes nouvelles de protection des plantes. Il n'a pas pour but de réaliser une synthèse objective de l'ensemble de la littérature disponible sur le sujet, mais plutôt de proposer une vision personnelle - et à ce titre certainement biaisée - des difficultés conceptuelles et pratiques auxquelles se trouve confronté le scientifique lorsqu'il veut favoriser l'adoption par le plus grand nombre possible d'utilisateurs des innovations techniques qu'il développe ou améliore. En ce sens, cet article n'a pas d'autre ambition que d'inciter le lecteur à réfléchir, à la lumière de sa propre expérience et de sa propre grille d'analyse, sur les facteurs qui déterminent le succès ou non d'une solution, validée scientifiquement, apportée à un problème porteur de forts enjeux collectifs.

Pour tenter de rendre le sujet moins aride, je l'ai traité ici comme un scénario d'enquête autour d'un exemple, la lutte contre Phytophthora infestans, agent du mildiou, par l'emploi des résistances variétales, que je crois représentatif d'un ensemble plus vaste de solutions biotechniques pouvant être apportées à des problèmes aussi lourds que la gestion des intrants en agriculture. Une démonstration similaire pourrait en effet être entreprise sans difficulté particulière en remplaçant "variété résistante" par "solution tech- nique innovante" - par exemple, par "lutte biologique" ou "stimulation des réactions de défense naturelle ", pour un grand nombre de couples plante/agent pathogène. N'étant ni économiste, ni sociologue, je n'aurai pas l'imprudence de me hasarder très avant sur des terrains que je maitrise mal : aussi, je me concentrerai ici sur l'une des solutions techniques que le chercheur offre au producteur, la culture de variétés résistantes, sur certains facteurs critiques déterminant (ou non) son adoption, et sur les conséquences que ceux-ci impliquent dans la conception et la réalisation des opérations de recherche.

\section{Constat de départ: utiliser des variétés résistantes, un acte rare}

Utiliser des variétés résistantes semble $a$ priori une solution de bon sens que la plupart des producteurs devraient employer. En effet, ces variétés sont à même de préserver le potentiel de production de la culture avec pas ou peu d'intrants pesticides, et donc sans atteinte à l'environnement, à la santé du consommateur ou à celle du producteur. Toutefois, la figure 1 montre que ce n'est pas le cas pour les résistances au mildiou en France, puisque la part occupée en culture par des cultivars sensibles (note inférieure ou égale à 4 sur l'échelle du CTPS [Comité technique permanent pour la sélection]) est sensiblement plus forte que la part de ces variétés au sein du catalogue officiel des variétés. Ceci explique en partie que le coût de la lutte contre le mildiou reste remarquablement élevé, de même que le nombre d'applications pesticides dirigées contre ce parasite (Johnson et al., 2000).

Cette difficulté des cultivars résistants à s'imposer en pratique n'est d'ailleurs pas limitée au seul cas du mildiou et de la France, mais existe aussi pour des productions très différentes comme celle des céréales (Vanloqueren et Baret,

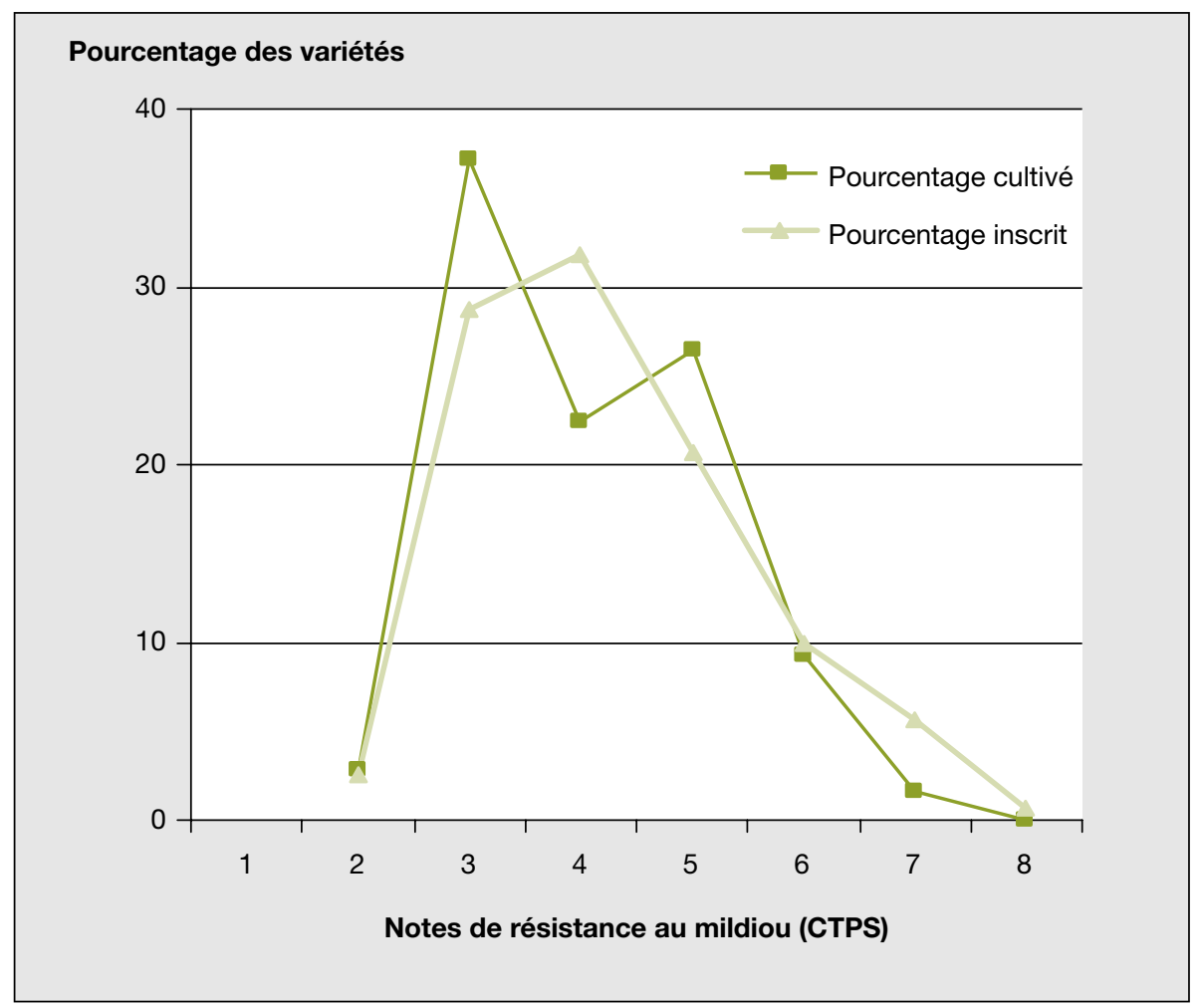

Figure 1. Proportion des variétés de pommes de terre, par classes de résistance au mildiou (1- très sensible à 9- très résistante), dans le catalogue officiel français des variétés (courbe en vert clair) et en culture de plant (courbe en vert sombre); données 2004.

Figure 1. Distribution of potato cultivars, according to their late blight resistance score in registration trials (1- highly susceptible to 9 - highly resistant), in the French official catalogue (in light blue) and in seed potato crops (in dark green) (2004 data). 
2008). Ces auteurs identifient 13 pistes, techniques, économiques ou sociologiques, susceptibles d'expliquer ce faible taux d'adoption. La suite de cet article examinera plus spécifiquement trois d'entre elles, qui me semblent particulièrement importantes dans une perspective d'élaboration de programmes de recherche : l'inadaptation des variétés résistantes par rapport aux systèmes de production dominants en Europe occidentale; un éventuel déficit de connaissance scientifique sur le fonctionnement des résistances elles mêmes, empêchant leur exploitation optimale; et le fait que la diffusion du progrès génétique ne cible pas suffisamment les véritables décideurs du choix variétal.

\section{Première piste :}

\section{la résistance, inadaptée aux systèmes actuels de production?}

Cette piste peut se décliner en deux jeux de questions: les résistances variétales sont-elles assez efficaces (autrement dit, le progrès génétique est-il suffisant?) ; et ces résistances sont-elles assez durables (autrement dit, les populations parasites s'y adaptent-elles suffisamment lentement)? Y répondre suppose d'évaluer les caractéristiques des résistances par rapport aux contraintes liées aux objectifs de production.

\section{Efficacité et progrès génétique}

D'après les données du Centre international de la pomme de terre ${ }^{1}$, la pomme de terre est aujourd'hui la troisième production alimentaire végétale mondiale, et la première non céréalière; sa production augmente régulièrement, en particulier en zone intertropicale et dans les pays à forte démographie (Inde, Chine, Asie du Sud Est). À part dans l'Amérique andine, où sont cultivées sept espèces différentes de Solanum tubérifères (Hawkes, 1990), seule la sous-espèce tétraplö̈de Solanum tuberosum tuberosum est utilisée. Les efforts de création variétale, soutenus

\footnotetext{
1 www.cipotato.org
}

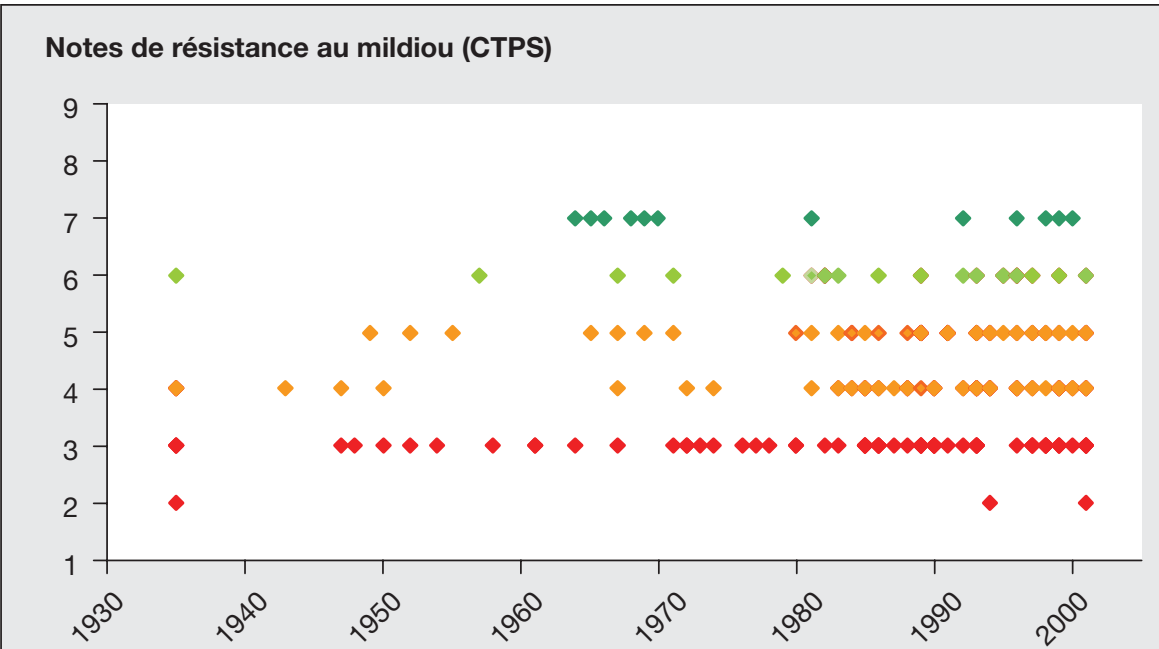

Années d'inscription

Figure 2. Distribution de sensibilité au mildiou (essais officiels du Comité technique permanent de la sélection [CTPS] ; 1: très sensible, à 9 : très résistante) des variétés de pommes de terre en fonction de leur année d'inscription.

Figure 2. Distribution of late blight resistance scores in official registration trials (1: highly susceptible to 9: highly resistant) of potato cultivars according to their date of registration.

depuis l'introduction de la pomme de terre en Europe (Salaman, 1985), ont généré de très nombreux cultivars's, tous multipliés par voie strictement végétative et représentant une large gamme de variabilité des traits agronomiques (précocité, résistance à divers bioagresseurs, rendement...) et d'utilisation (couleur, forme et composition du tubercule...).

Deux types principaux de résistance existent vis-à-vis de $P$. infestans chez la pomme de terre. Les gènes majeurs (ou gènes $\mathrm{R}$ ) fonctionnent selon le modèle "gène pour gène" de Flor (1956), et confèrent une résistance totale (absence d'extension des symptômes et de multiplication du parasite) vis-à-vis des isolats dépourvus des allèles de virulence correspondants. Sur le plan épidémiologique, ces résistances se traduisent par un décalage dans le temps du début de l'épidémie par rapport aux variétés sensibles (Vanderplank, 1968); cette caractéristique peut d'ailleurs être utilisée pour reconnâtre la présence de gènes majeurs à partir de l'observation de la progression épidémique au champ (Andrivon et al., 2006). Pour leur part, les résistances quantitatives partielles réduisent la taille et la vitesse de croissance des symptômes, et/ ou diminuent le taux de multiplication du

\footnotetext{
$\overline{2}$ II en existe plus de 500 actuellement répertoriés sur la base de données Europotato.org.
}

parasite par rapport à une infection sur cultivar sensible (Vanderplank, 1968), mais souvent de manière insuffisante en cas de forte pression parasitaire (Wastie, 1991). Elles sont généralement sous déterminisme polygénique, des $\mathrm{QTL}^{3}$ de résistance ayant pu être localisés sur chacun des 12 chromosomes que compte le génome haploïde de $S$. tuberosum (voir, par exemple, Simko, 2002). Les deux types de résistance peuvent être rencontrés, séparément ou combinés, chez les cultivars commerciaux ; la plupart de ces cultivars sont néanmoins dépourvus de gènes $\mathrm{R}$ efficaces, et possèdent des niveaux faibles à modérés de résistance quantitative, leur conférant une résistance au champ limitée (figure 1).

L'examen du niveau de résistance au mildiou des variétés de pomme de terre inscrites au catalogue français depuis sa création dans les années 1930 ne montre pas de tendance claire et continue à l'amélioration de la performance variétale vis-à-vis de ce parasite (figure 2). Certes, des variétés à bon niveau de résistance sont régulièrement inscrites, et ce dans tous les types variétaux. Toutefois, nombre de variétés sensibles, voire très sensibles, mais dotées d'autres qualités (saveur, rendement, qualité culinaire ou

\footnotetext{
${ }^{3}$ Quantitative trait locus
} 
de transformation...) trouvent aussi leur place dans le catalogue, y compris au cours des années les plus récentes. Si l'on rapproche ce constat de celui de la prédominance des variétés sensibles en culture, on est fondé à penser soit que la résistance au mildiou n'est pas un critère central dans l'acceptation d'une variété, soit que les niveaux de résistance actuels restent insuffisants pour convaincre les utilisateurs de modifier leur choix variétal sur la base de ce seul critère.

\section{Adaptation des populations et stabilité des résistances}

Le constat précédent peut sembler sévère au vu des efforts déployés par les obtenteurs en matière de résistance. Il doit néanmoins être mis en perspective. En effet, la performance d'une variété au moment de son inscription ne préjuge pas de sa stabilité au cours du temps. Il se trouve que les variétés ayant jusqu'à présent obtenu les notes les plus élevées dans les épreuves d'inscription devaient ce succès à la présence d'un ou de plusieurs gènes majeurs, rapidement surmontés lors du déploiement de ces variétés à plus grande échelle (Pilet et al., 2005, Montarry et al., 2006).

Cette instabilité des résistances les plus fortes dissuade probablement beaucoup d'utilisateurs d'accorder un poids sensible à ce critère lors des choix variétaux. Une caractéristique importante de la culture de pomme de terre dans la plupart des pays est en effet la tendance à la concentration variétale au sein de bassins de production. Ceci tient à la fois au mode de multiplication végétative de la plante, à la difficulté du transport des tubercules poids et volume importants, richesse en eau - et à la spécialisation des productions pour des marchés locaux - préférences alimentaires locales; présence d'une usine de transformation ou de conditionnement. Il en résulte que la plupart des régions constituent des "quasi-monocultures " d'un seul cultivar, et ce sur des périodes étendues dans le temps. L'exemple extrême est l'île de Jersey, où une seule variété ("International Kidney", localement connue sous le nom de "Jersey Royal ") occupe plus de $95 \%$ des surfaces et est cultivée depuis plus de 120 ans.

Cela donne aux parasites de très grandes opportunités d'adaptation, et donc une forte probabilité de surmonter les résistances déployées (Marshall, 1977). Dans le cas précis qui nous occupe ici, cette adaptation est favorisée par plusieurs caractéristiques du cycle de vie de $P h y$ tophthora infestans. Ce parasite est en effet capable d'infecter tous les organes de la pomme de terre (feuillage, tiges, tubercules, et même les racines), et a développé un mode de vie quasi strictement biotrophe in natura (Andrivon, 1995). Ce mode de vie, conjugué au caractère polycyclique des épidémies, induit une relation très étroite du parasite avec son hôte et donc une capacité de réponse rapide aux pressions sélectives (gènes de résistance en particulier) que celui-ci lui impose. Le fort potentiel adaptatif qui en découle se manifeste régulièrement par le contournement, en quelques saisons culturales, des nouveaux gènes majeurs déployés en culture (Wastie, 1991 ; Pilet et al., 2005 ; Fry, 2008), mais aussi par la possibilité d'érosion de résistances quantitatives partielles (Andrivon et al., 2007).

L'étude de populations du parasite confrontées à des variétés portant différents types ou niveaux de résistance a montré que ces populations peuvent être structurées par les variétés hôtes pour les composantes quantitatives du pouvoir pathogène. Toutefois, dans ce cas, le déterminant principal de l'adaptation est la dominance de la variété dans le paysage variétal, plus que son niveau de résistance (Flier et al., 2007 ; Montarry et al., 2006, 2008). L'échelle spatiale est donc importante pour la mise en évidence de tels patrons adaptatifs, le facteur clé pour la différenciation entre populations semblant être l'absence effective de flux de gènes (Andrivon et al., 2007 ; Montarry et al., 2008). Il est ainsi possible d'observer une adaptation aux variétés dominantes, qu'elles soient initialement sensibles ou partiellement résistantes au parasite, à des échelles géographiques suffisantes pour empêcher les échanges génétiques entre populations sélectionnées sur différents génotypes hôtes.

\section{Deuxième piste :} nos connaissances scientifiques

\section{sont-elles suffisantes?}

Il découle des observations précédentes que le type de résistance (en particulier le caractère quantitatif ou partiel de la résistance) ne confère pas par lui-même de garantie de durabilité accrue: en résumé, (presque) toutes les résistances s'usent quand on s'en sert (beaucoup). Une gestion, dans le temps et dans l'espace, du déploiement de chaque résistance, est donc nécessaire pour augmenter la durabilité de ces ressources génétiques vulnérables (Pink, 2002 ; McDonald et Linde, 2002). Cette gestion doit être prise en compte dès la construction des génotypes résistants : elle concerne des temps longs et des échelles spatiales vastes ; aussi, sa validation a priori n'est pas accessible à l'expérimentation. Cela qui rend indispensable le recours à la modélisation. Néanmoins ces modèles ne peuvent être construits qu'à partir de connaissances préalablement acquises. Il est donc légitime de s'interroger sur la disponibilité de ces connaissances.

Beaucoup de choses ont été écrites sur le mildiou et son agent, depuis les premières grandes épidémies européennes des années 1845-1850. La littérature scientifique concernant les résistances est un peu plus récente, les premiers travaux en ce sens remontant au début du $\mathrm{xx}^{\mathrm{e}}$ siècle (cf Wastie, 1991 pour une synthèse critique). Les activités de recherche des 160 dernières années ont ainsi généré un corpus de connaissances considérables sur la maladie et son agent, ainsi que sur les caractéristiques : des résistances disponibles chez l'hôte (déterminisme génétique, mécanismes biochimiques et moléculaires, etc. Fry, 2008).

Toutefois, seule une très faible partie des études publiées concerne l'adaptation du parasite à la résistance de l'hôte (virulence et agressivité, spécificité isolat/ population, tempo et échelle de l'adaptation, etc.) et surtout la manière de combiner la résistance avec d'autres méthodes de lutte. Ainsi, une recherche dans la base de données ISI Web of Science sur les mots clés "Phytophthora infestans" ou "late blight" fournit 265 articles publiés entre 2000 et 2004 ; seuls 33 d'entre eux (soit $12 \%$ ) traitaient de méthodes de lutte, dont seulement 9 (soit un fort maigre 3,3\% du total) étaient consacrés à l'utilisation de cultivars résistants, seuls ou en combinaison avec d'autres méthodes de lutte. Dans cette même base, seule une quinzaine d'articles, sur les 143 centrés sur le parasite lui-même, étaient consacrés à la distribution et à l'évolution des caractères de pouvoir pathogène au sein des populations de $P$. infestans. Par comparaison, les articles traitant des aspects génétiques, génomiques et mécanistiques 
de la résistance au mildiou représentaient $34 \%$ du corpus total, soit 89 articles.

Cette analyse sommaire suggère deux conclusions. Primo, les fronts de science actuellement explorés dans un cas comme celui du mildiou de la pomme de terre portent essentiellement sur la connaissance du fonctionnement moléculaire des interactions, sans que la manière de prendre en compte ces connaissances pour la mise au point de solutions techniques au problème agronomique lui-même (limiter les attaques de mildiou et/ou les pertes qui leur sont imputables) soit véritablement à l'ordre du jour. Secundo, les efforts pour construire ou pour valider, à partir de jeux de données réelles, les systèmes maximisant la durabilité des résistances reposent sur une approche aggrégative et combinatoire à partir d'éléments étudiés initialement séparément, et très rarement sur une analyse réellement systémique, à savoir l'identification des performances attendues du système, des verrous et donc des leviers à actionner simultanément.

\section{Troisième piste: les producteurs, acteurs ou victimes?}

Identifier des solutions biologiquement pertinentes pour exploiter les résistances les plus efficaces et réduire l'adaptation des populations est une étape nécessaire. Elle n'est toutefois pas suffisante, l'acceptabilité pratique de ces solutions devant encore être éprouvée. Ainsi, divers travaux (Andrivon et al., 2003; Garrett et Mundt, 2000 ; Phillips et al., 2005 ; Pilet et al., 2006) ont montré qu'associer des variétés possédant des résistances différentes au sein d'une même parcelle permet de réduire la sévérité des épidémies de mildiou, dans des proportions parfois importantes; il en va de même pour des associations interspécifiques impliquant des plantes non hôtes (Bouws et Finckh, 2008). L'acceptabilité de tels schémas de diversification, bonne dans certaines situations - lorsque les circuits de commercialisation sont courts, comme c'est le cas dans beaucoup d'exploitations en agriculture biologique -, exige toutefois des modifications de pratiques ou une augmentation des coûts de production parfois rédhibitoires (Wolfe, 1985).
La mise en œuvre des méthodes de protection des plantes fait intervenir une grande diversité de protagonistes humains: chercheurs, prescripteurs, développeurs, commerçants ou producteurs. Tous ont une part de responsabilité et d'initiative dans le choix, l'évaluation ou la concrétisation des actions, au sein d'une chaine d'interactions souvent complexe. Les chercheurs (ceux du secteur public comme du secteur privé) sont à l'origine des outils entrant dans ces méthodes de phytoprotection (variétés résistantes, molécules pesticides ou agents biologiques, appareillage, systèmes d'aide à la décision...). Ils jouent également un rôle important, mais discret, dans la prescription elle-même, en particulier quand celle-ci porte sur des choix stratégiques plutôt que tactiques - l'intervention sur les systèmes de production, par exemple. La spécialisation des chercheurs tend toutefois à leur faire perdre de vue la globalité du contexte économique, technique et social, dans lequel les outils qu'ils produisent doivent trouver place, et les contraintes qui en découlent. Ce décalage est fréquemment à l'origine de l'incompréhension mutuelle entre acteurs et de la proportion finalement faible des innovations issues de la recherche qui débouchent à court terme sur une application effective dans la pratique agricole. De là provient une grande part de la frustration réciproque entre des praticiens jugeant la recherche " trop fondamentale " pour leurs besoins et des chercheurs qui s'étonnent du peu de succès des innovations qu'ils proposent.

\section{Identifier les vrais décideurs}

En France, environ $60 \%$ des pommes de terre de consommation, et près de $100 \%$ de celles qui sont destinées à la transformation industrielle, sont cultivées sous contrat (industries de transformation, supermarchés, distributeurs grossistes). Les producteurs fournissent alors les surfaces, les machines et la force de travail, mais ils sont rarement décisionnaires sur des domaines stratégiques comme le choix variétal, imposé par l'acheteur. Un corollaire immédiat de cette situation est que la diversification variétale et le recours plus systématique à des cultivars résistants ne sera possible, pour des productions à forte composante contractuelle, que si ces cultivars satisfont les besoins techniques, mais aussi la stratégie commerciale des acheteurs. La tendance actuelle au développement de contrats d'exclusivité pour les variétés nouvelles, voire au développement de partenariats étroits entre industriels et obtenteurs pour des programmes de création variétale dédiés, va incontestablement dans ce sens, de même que la multiplication des labels, appellations d'origine contrôlée (AOC) ou cahiers des charges spécifiques à l'une ou l'autre enseigne. Dans ce contexte, il est donc essentiel que la communication sur les acquis en matière de gestion spatiale et temporelle des ressources génétiques cible autant (si ce n'est plus) les acheteurs que les producteurs eux-mêmes. Le développement commercial des cultivars de pomme de terre est en effet un processus lent, et les modifications du paysage variétal pour cette espèce sont infiniment moins rapides que pour d'autres grandes cultures annuelles (céréales ou colza, par exemple).

La contractualisation croissante des productions industrielles, si elle transfère l'initiative de la décision du producteur à l'acheteur, n'en est pas moins régie par un cadre réglementaire de plus en plus présent. C'est donc non seulement les acheteurs, mais aussi les politiques qu'il est important de convaincre de la viabilité de ces solutions complexes, mais durables. On peut ainsi noter que les récentes décisions politiques (par exemple, la directive européenne sur l'utilisation des produits phytosanitaires, qui prescrit le passage de l'ensemble de l'agriculture européenne aux pratiques de la protection intégrée d'ici à 2014, ou le Plan interministériel français de réduction des pesticides, qui prévoit une diminution de $50 \%$ des applications de pesticides à l'échéance 2018), sont certainement de nature à favoriser l'adoption de méthodes jusque là marginales, car trop peu efficaces par rapport aux applications phytosanitaires (Aubertot et Barbier, 2005).

\section{Prendre en compte les contraintes des acteurs}

La production de pommes de terre exige des investissements spécifiques - machines propres à cette culture (comme les planteuses ou arracheuses, les tables de tri, etc.) ; installations de stockage ventilées et/ou réfrigérées -, qui imposent une spécialisation des producteurs. Elle concerne de plus en plus des exploitations de grande taille, qui emploient peu de main d'œuvre. Sur de telles exploitations, où la technicité est élevée, mais où l'organisation du travail est une contrainte forte, il est plus facilement gérable pour le chef d'entreprise de réaliser un planning 
d'interventions (par exemple l'application hebdomadaire d'un pesticide) que de piloter ces interventions sur la base de systèmes d'aide à la décision dépendant d'un contexte météo difficile à anticiper. Ceci favorise l'application de type " assurance " de pesticides bon marché, plutôt qu'une optimisation des passages en fonction du risque constaté sur la parcelle ou prévu par un modèle de simulation. La pratique de tels traitements d'assurance est d'autant plus importante aujourd'hui en production de pommes de terre que nombre de matières actives, peu remobilisées dans la plante ${ }^{4}$, sont à efficacité préventive, et que le coût des applications reste faible par rapport au risque de perte totale ou partielle d'une culture de rente en cas de développement d'une épidémie.

\section{Épilogue}

\section{- variétés résistantes, pathologistes \\ et protection intégrée : quel avenir?}

Les phytiatres ont encore beaucoup à offrir pour l'élaboration de stratégies de protection intégrée adaptées aux besoins d'une "agriculture écologiquement intensive". Ces contributions possibles peuvent se décliner selon au moins trois directions, complémentaires et toutes nécessaires. La première est le développement de nouveaux outils de gestion des épidémies, comme, par exemple, des cultivars résistants ou de nouveaux idéotypes variétaux, incorporant à la fois des résistances à l'infection et à l'invasion, mais aussi des traits contribuant à l'évitement des infections (architecture des plantes et des couverts, et asynchronie des cycles de sensibilité de la plante et de présence du bioagresseur). La deuxième est la prise en compte des échelles spatiales et temporelles de déploiement, comme cela a été tenté par le passé avec les schémas de diversification variétale (Wolfe et Schwarzbach, 1978) ou les associations variétales. La troisième est de produire des modèles opéra-

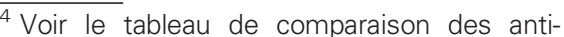
mildious sur http://www.euroblight.net/Fungicide/FungicideComparison. asp?language $=$ UK.
}

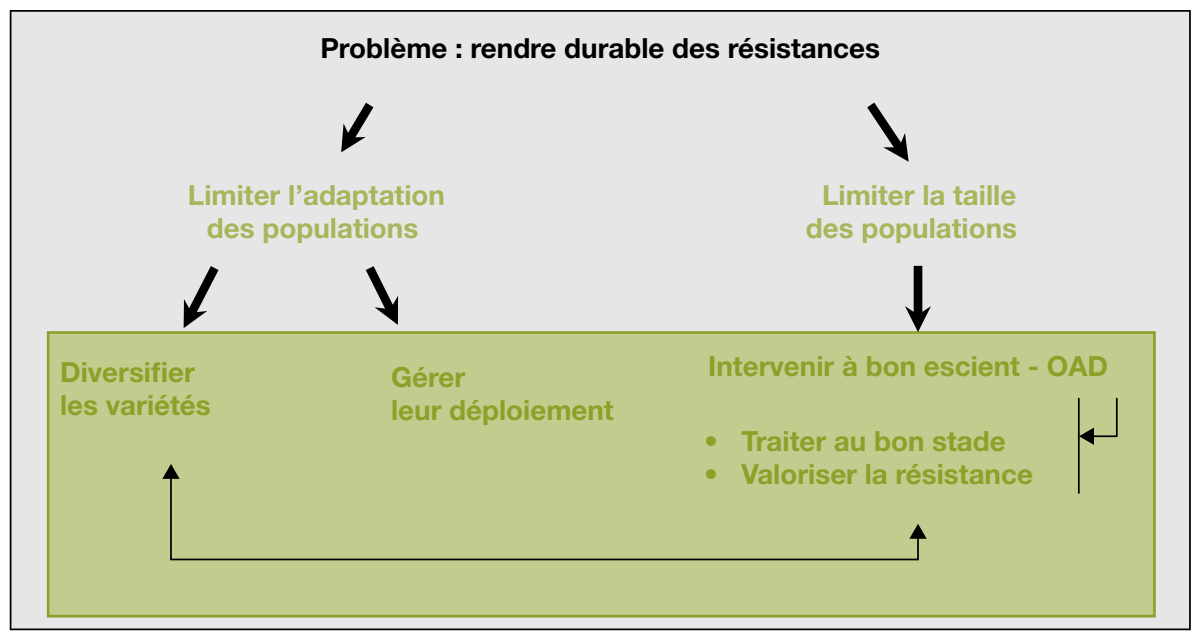

Figure 3. Représentation schématique des éléments à intégrer pour une gestion durable des résistances variétales.

Figure 3. Diagrammatic representation of elements to be integrated for the sustainable management of host resistance genes.

$O A D$ : outils d'aide à la décision.

tionnels de gestion variétale, permettant d'intégrer idéotypes résistants et stratégies de déploiement dans une optique prédictive - en d'autres termes, proposer des modes d'emploi avec les variétés. Si nous sommes encore loin de cet objectif, en particulier pour les résistances quantitatives, celui-ci reste atteignable, en particulier grâce aux développements de la sélection assistée par marqueurs, qui permet de mieux contrôler la construction des génotypes, et aux avancées en écologie évolutive des populations de bioagresseurs, avancées qui proposent des clés d'anticipation des réponses adaptatives de ces populations à l'emploi des hôtes résistants. L'exemple des systèmes de gestion pour des résistances aux insectes introduites par transgénèse (Gould, 1998) est ainsi un prototype de tels déploiements "sur mesure "d'hôtes résistants.

Si la compréhension des dynamiques adaptatives et le développement de nouveaux idéotypes variétaux sont nécessaires, ils doivent être complétés par une appréhension globale de la place de ces modes de gestion des épidémies dans les systèmes de production. Il ne s'agit pas de changer simplement d'arme, mais bien de modifier les modes de pensée et de prise de décision. En effet, l'objectif d'une agriculture durable, c'est-à-dire assurant un revenu suffisant aux producteurs tout en préservant l'environnement et les ressources, ne peut être atteint en recourant uniquement à des solutions tactiques interchangeables (dois-je traiter aujourd'hui ou pas ?); il suppose au contraire une gestion stratégique (dois-je utiliser des variétés résistantes et, si oui, avec quelles mesures d'accompagnement?), comme le montre le prototype de gestion intégrée illustré par la figure 3. Un des points d'interrogation clés est donc de savoir dans quelle mesure ces choix stratégiques de moyen ou long terme sont compatibles avec les modes actuels de prise de décision, souvent axés sur le court terme et pilotés par les prix (fluctuants) de marché. Un autre élément d'interrogation est de savoir si les phytiatres (chercheurs, techniciens et prescripteurs) évoqués plus haut sont en mesure de fournir l'information requise quant à l'efficacité, la complémentarité ou la compatibilité des différentes mesures à mettre en œuvre au sein de systèmes de protection et de production intégrés. Enfin, il est impératif d'adapter les armes -et les efforts de communication - aux cibles, c'est-à-dire aux véritables décideurs. Ce n'est qu'ainsi que les "truands " permettront enfin aux "bons " de triompher durablement des "brutes".

\section{Remerciements \\ Les idées exposées dans cet article doi- vent beaucoup à des discussions tenues au cours des 15 dernières années avec de nombreux acteurs de la protection des plantes: Serge Duvauchelle, Christian Lannou, Daniel Ellissèche et Serge Savary et leurs apports m'ont été particulière- ment précieux. Je tiens à remercier ici}


Marie-Claude Roland (Inra, Linguistique et pratiques de recherche), Hélène Magalon et Hortense Brun, ainsi que les trois lecteurs experts anonymes, pour leurs remarques constructives sur le manuscrit.

\section{Références}

Andrivon D. Biology, ecology and epidemiology of the potato late blight pathogen Phy tophthora infestans in soil. Phytopathology $1995 ; 85: 1053-6$.

Andrivon D, Lucas JM, Ellissèche D. Development of natural late blight epidemics in pure and mixed plots of potato cultivars with different levels of partial resistance. Plant Pathol $2003 ; 52$ : 586-94.

Andrivon D, Pellé R, Ellissèche D. Assessing resistance types and levels to epidemic diseases from the analysis of disease progress cur ves: principles and application to potato late blight. Am J Potato Res 2006 ; 83 : 455-61.

Andrivon D, Pilet F, Montarry J, et al. Adaptation of Phytophthora infestans to partial resistance in potato: evidence from French and Moroccan populations. Phytopathology 2007 $97: 338-43$.

Aubertot JN, Barbier JM, A. Carpentier A, et al Pesticides, agriculture et environnement. Réduire l'utilisation des pesticides et limiter leurs impacts environnementaux. Rapport d'expertise scientifique collective. Paris : Inra Cemagref, 2005.

Bouws H, Finckh MR. Effects of strip intercropping of potatoes with non-hosts on late blight severity and tuber yield in organic production. Plant Pathol 2008 ; 57 : 916-27.
Flier WG, Kroon LPNM, Hermansen A, et al. Genetic structure and pathogenicity of populations of Phytophthora infestans from organic potato crops in France, Norway, Switzerland and the United Kingdom. Plant Pathol 2007; $56: 562-72$.

Flor $\mathrm{HH}$. The complementary genetic systems in flax and flax rust. Adv Genet 1956; 8: 29-54.

Fry WE. Phytophthora infestans: the plant (and R gene) destroyer. Mol Plant Pathol 2008; 9 : $385-402$.

Garrett KA, Mundt CC. Host diversity can reduce potato late blight severity for focal and general patterns of primary inoculum. Phytopathology $2000 ; 90$ : 1307-12.

Gould F. Sustainability of transgenic insecticidal cultivars: integrating pest genetics and ecology. Annu Rev Entomol 1998; 43 : 701-26.

Hawkes JG. The potato - evolution, biodiversity \& genetic resources. Londres: Belhaven Press, 1990.

Johnson DA, Cummings TF, Hamm PB. Cost of fungicides used to manage potato late blight in the Columbia Basin: 1996 to 1998. Plant Dis $2000 ; 84: 399-402$.

Marshall DR. The advantages and hazards of genetic homogeneity. Proc New York Acad Sci $1977 ; 287: 1-20$.

McDonald BA, Linde C. Pathogen population genetics, evolutionary potential and durable resistance. Annu Rev Phytopathol 2002; 40 : 349-79.

Montarry J, Corbière R, Lesueur S, et al. Does selection by resistant hosts trigger local adaptation in plant-pathogen systems? J Evol Biol $2006 ; 19: 522-31$

Montarry J, Glais I, Corbière R, et al. Adaptation to the most abundant host genotype in an agricultural plant-pathogen system - potato late blight. J Evol Biol 2008; 21 : 1397-407.
Pilet $F$, Pellé $R$, Ellissèche $D$, et al. Efficacy of the $\mathrm{R} 2$ resistance gene as a component of durable management of potato late blight in France. Plant Pathol 2005 ; 54 : 723-32.

Pilet F, Chacón M, Forbes GA, et al. Protection of susceptible potato cultivars against late blight in mixtures increases with decreasing inoculum pressure. Phytopathology 2006; 96 : 777-83.

Pink DAC. Strategies using genes for nondurable disease resistance. Euphytica 2002 ; $124: 227-36$.

Phillips SL, Shaw MW, Wolfe MS. The effect of potato variety mixtures on epidemics of late blight in relation to plot size and level of resistance. Ann Appl Biol 2005 ; 147 : 245-52.

Salaman RN. The history and social influence of the potato. Revised impression, edited with a new introduction by J.G. Hawkes. Cambridge (Royaume-Uni): Cambridge Univ. Press, 1985.

Simko I. Comparative analysis of quantitative trait loci for foliage resistance to Phytophthora infestans in tuber-bearing Solanum species. Am J Potato Res 2002 : 79: 125-32.

Vanderplank JE. Disease resistance in plants. New-York (USA) : Academic Press, 1968.

Vanloqueren G, Baret PV. Why are ecological, low-input, multi-resistant wheat cultivars slow to develop commercially? A Belgian agricultural 'lock-in' case study. Ecol Econ 2008; 66 : 436-46.

Wastie RL. Breeding for resistance. In : Ingram DS, Williams PH, eds. Phytophthora infestans, the cause of late blight of potato (Advances in Plant Pathology, vol. 7). London: Academic Press, 1991.

Wolfe MS. The current status and prospects of multiline cultivars and variety mixtures for disease resistance. Annu Rev Phytopathol $1985 ; 23: 251-73$.

Wolfe MS, Schwarzbach E. Patterns of race changes in powdery mildews. Annu Rev Phy topathol $1978 ; 16: 159-80$. 